\title{
A Novel Approach to Use ZnO Thin Film as a Switching in Dynamic Random Access Memory (DRAM) Cell
}

\author{
Amit Kumar \\ Department of \\ computer science, \\ Institute of Engineering \\ \& Technology, North \\ Extn., M.I.A. Alwar- \\ 301030, Rajasthan, \\ India
}

\author{
Deepak chaudhary \\ Department of \\ computer science, \\ Institute of \\ Engineering\& \\ Technology, North \\ Extn, M.I.A. Alwar- \\ 301030, Rajasthan, \\ India
}

\author{
Manoj Kumar \\ Department of \\ Electrical, Electronic \& \\ Information \\ Engineering, \\ Toyohashi University of \\ Technology, Tempaku- \\ cho, Hibariga-oka1-1, \\ Toyohashi-441-8580, \\ Japan
}

\author{
Beer Pal Singh \\ Department of Physics, Ch. \\ Charan Singh University, \\ Meerut - 250 004, U. P. India
}

\begin{abstract}
Resistance switching random access memory (RRAM) has drawn considerable attention for the application in non- volatile memory element in semiconductor memory devices. A $\mathrm{ZnO}$ thin film now assumed to be useful for dynamic random access memory (DRAM) cell. In this paper we provide a framework to its use as a switching ON or OFF in DRAM cell. In this type of memory cell the $\mathrm{ZnO}$ thin film has a lot of importance instead of a transistor. Inside the DRAM cell, we are suggested to use the $\mathrm{ZnO}$ thin film due to its reliable and repeated switching of the resistance. Thus after the replacement of $\mathrm{ZnO}$ thin film as a switching element instead of a transistor, the DRAM cell has a strong resistance switching capability.
\end{abstract}

\section{Keywords}

ZnO thin film, Resistance switching, DRAM

\section{INTRODUCTION}

There has been much interest seeking a high-density, highspeed, and low power memory technology that preserves its data even during power failed. Currently, resistance switching random access memory phenomena has paid great attention to study actively in order to apply them to the next generation nonvolatile memory devices. [1-10]. Advanced research is in progress to find alternative materials for non volatile memories focus on accomplishing greater packing density, faster switching rate, lower power consumption and low voltage operation compared to commonly used MOS transistor based flash memory device. Resistive switching characteristics of multilayered $\left(\mathrm{HfO}_{2} / \mathrm{Al}_{2} \mathrm{O}_{3}\right)$ n thin film was recently studied by W. H. Tzeng et.al [11]. Some of the currently growing ideas in this area are silicon oxide nitride oxide silicon based memory (SONOS), magneto-resistive random access memory (MRAM), phase change random access memory (PRAM) and resistive random access memory (RRAM). The simple phenomenon of resistance switching of RRAM observed in the specific form of the thin films of the metal oxide such as $\mathrm{ZrO}_{2}, \mathrm{TiO}_{2}, \mathrm{NiO}$, $\mathrm{MnO}_{2}$ and recently $\mathrm{ZnO}$ [12-15]. Amongst the known metal oxides currently being explored for the development of RRAM, $\mathrm{ZnO}$ has been demonstrated to be a potential candidate due to its outstanding features such as high exciton binding energy $\sim 60$ $\mathrm{meV}$. This excitation stability provides opportunities for making highly efficient device at room temperature. $\mathrm{ZnO}$ has a direct and wide band gap of $3.4 \mathrm{eV}$ at room temperature and it is relatively hard material and has high melting temperature of about $2000{ }^{\circ} \mathrm{C}$, its hardness could conceivably expand the life time of devices. $\mathrm{ZnO}$ cross-bar array resistive random access memory stacked with heterostructure diodes for eliminating the sneak current effect was studied by J. W. Seo et. al [16]. J. Qiu et. al

[17] has observed the effects of metal contacts and dopants on the performance of $\mathrm{ZnO}$-based memristive devices.

In this paper, we provide the brief knowledge of the introduction, application and resistance switching (RS) properties of the $\mathrm{ZnO}$ thin film. The next section of the paper provides the assumed effect of the $\mathrm{ZnO}$ thin film in the DRAM cell.

\section{ZnO THIN FILM AND ITS USE}

$\mathrm{ZnO}$ is naturally an n-type semiconductor. The intrinsic defect levels that lead to n-type behavior lay approximately $0.01-0.05$ $\mathrm{eV}$ below the conduction band. Zinc oxide thin films can retain the hexagonal wurtzite structure and are composed of columnar crystallites with grain sizes in the range of 50-600 $\AA$. $\mathrm{ZnO}$ thin films can grow in polycrystalline and epitaxial nature. The grown films show a strong c-axis orientation perpendicular or parallel to the substrates and the degree or preferred orientation depends on the substrate material and deposition parameters $[18,19]$. However, epitaxial growth of $\mathrm{ZnO}$ thin films on different lattice matched substrates has been reported by Kucheyev et. al $[20,21]$ and has shown huge improvement in crystalline quality and enhanced electrical and optical properties.

The undoped $\mathrm{ZnO}$ thin films exhibits a high transmittance (about $85-90 \%$ ) in the visible region which falls sharply in the UV region because of the one set of fundamental absorption and gives highest reflectance (about 5-15\%) in the visible region. $\mathrm{ZnO}$ films can be produced by $\mathrm{DC}$ reactive and magnetron sputtering $[22,23]$, metal-organic chemical vapor deposition (MOCVD) [24, 25], vacuum evaporation [26], solgel method [27], pulsed laser deposition [28] and spray pyrolysis [29]. The grown films exhibit high crystalline quality along with preferred c-axis orientation. Undoped $\mathrm{ZnO}$ thin films reveal a nearly constant transmittance up to $2.5 \mu \mathrm{m}$ and a nearly constant reflectance up to $10 \mu \mathrm{m}$. The band gap has been reported to vary slightly with annealing temperature and the refractive index varying from 1.7 to 1.91 [30] compared to bulk value of 2.1. The photoluminescence (PL) properties of $\mathrm{ZnO}$ thin films ascribed to strongly bound exciton state, and defect states. These defect states may be originated due oxygen deficiency or point defects [31]. A strong near band edge UV PL peak appeared at $3.2 \mathrm{eV}$ is attributed to an exciton state at room temperature, as the binding energy is of the order of 60 $\mathrm{meV}$. The PL spectrum at low temperature $(10 \mathrm{~K})$ exhibits more 
than one emission lines. The bound exciton emission lines at $3.356,3.360$ and $3.367 \mathrm{eV}$ dominate the PL spectrum of undoped $\mathrm{ZnO}$ films. These emission lines could be correlated with defect complexes. More emission lines are not useful for optoelectronic devices such as light emitting diode and laser diodes. The resistivity of undoped $\mathrm{ZnO}$ thin films has been reported by many researchers in the wide range in the order of $10^{-2}-10^{9} \Omega-\mathrm{cm}$. The conversion from conducting behavior to insulating behavior has been attributed to the unstable state of defects behaving as shallow donors in as grown films.

Most of the literatures reveal that the thin film technologies are also being developed as a means of substantially reducing the cost of electronic equipments and enhance the device performance. The nano structured thin film enhances the efficiency of solar cells, memory storage capacity of the computers, reduces the costs of the devices as well as the materials losses. Transparent conducting thin films continue to receive considerable attention from both fundamental and application point of view primarily because of their useful properties.

$\mathrm{ZnO}$ thin films generated immense interest as a transparent semiconducting material, because of coating of this material are relatively inexpensive. Unique properties of $\mathrm{ZnO}$ make it a very promising material for much diversified fields. It has II-VI compound semiconductor and is well known for its attractive features. It is attracting considerable attention, particularly in thin film form, for application in wide range of optoelectronic devices. Due to its low cost, non-toxicity, readily availability and high chemical stability even in reducing environments, $\mathrm{ZnO}$ has become most promising material for device applications. $\mathrm{ZnO}$ is extensively used in surface acoustic wave devices [32], gas sensors [33], varistors [34], and light modulators. It is very useful material for the industrial applications such as elecro photography, electroluminescence phosphorus, gas dector, medical and cosmetics and pigment in paints.

The electrical, structural and optical quality of $\mathrm{ZnO}$ thin films greatly enhanced in recent years, which has drawn considerable interest in the use of this material for short wavelength light emitters and transparent electronics. Highly conductive and transparent $\mathrm{ZnO}$ thin films could easily be grown with inexpensive deposition techniques. Preferred orientation of the film is very important to utilize for device applications. A preferred orientation is a special phenomenon in which certain crystal plane grows parallel to the substrate as is often observed in thin films prepared by different techniques. It is one of the most important properties in thin films both from theoretical and application point of view.

\section{EXPERIMENTAL DETAILS}

$\mathrm{ZnO}$ thin films on $\mathrm{Ti} / \mathrm{SiO}_{2} / \mathrm{n}$-Si were grown by $\mathrm{RF}$ magnetron sputtering technique. The experimental details can be found elsewhere [35]. The thickness of the resulting $\mathrm{ZnO}$ thin film was estimated to be around $150 \mathrm{~nm}$. Both top and bottom contacts were deposited using RF sputtering technique. The direction of current flow was kept from top to bottom electrode and the measurements were carried out in voltage sweeping mode.

\section{RESISTANCE SWITCHING PROPERTIES OF ZnO THIN FILM}

Polycrystalline $\mathrm{ZnO}$ thin films were grown highly resistive and preferred c-axis oriented by different deposition techniques for studied their resistive switching behaviour. The $\mathrm{ZnO}$ thin films resistance was observed to switch between a high and low resistance states [2]. Conduction behaviour was found to be dominated by ohmic behaviour in LRS (OFF state) and Poole Frenkel emission in HRS (ON state). The device based on $\mathrm{ZnO}$ thin film demonstrates reliable resistive switching properties such as low switching voltage and switching cycling. Figure 1 shows the Current-Voltage (I-V) plot $\mathrm{ZnO}$ thin film showing resistive switching [12], which indicate the two state one is in high resistance state and second in low resistance state (RESET $\&$ SET respectively) that is repeated over the no of cycles. This property of the $\mathrm{ZnO}$ thin film is very useful in the dynamic random access memory (DRAM) cell.

After the forming process on the pristine memory cell, the resistive memory switching is generally observed by a voltage sweep. As the applied voltage increases, the resistive transition from a stable low resistance $R_{\mathrm{ON}}$ state to a stable high resistance $R_{\mathrm{OFF}}$ state, the RESET, appears at $1.6 \mathrm{~V}$. At the $R_{\mathrm{OFF}}$ state, the resistive transition from $R_{\mathrm{OFF}}$ state to $R_{\mathrm{ON}}$ state, the SET, occurs by increasing the voltage up to $5 \mathrm{~V}$ as can be seen in the Fig. 1 . For the SET and the forming process, an appropriate current compliance should be imposed for the memory switching.

The current is limited by an external circuit, and the voltage sweep stops when the measured current reaches a current compliance value, which is experimentally performed. The voltage required for each transition is defined as the RESET voltage $\left(V_{\text {RESET }}\right)$ and the SET voltage $\left(V_{\mathrm{SET}}\right)$. As shown in Fig. 2 the voltage-biased SET inevitably results in a sudden jump of the measured current at $V_{\mathrm{SET}}$. This blurs the exact role of the external current at the SET transition point.

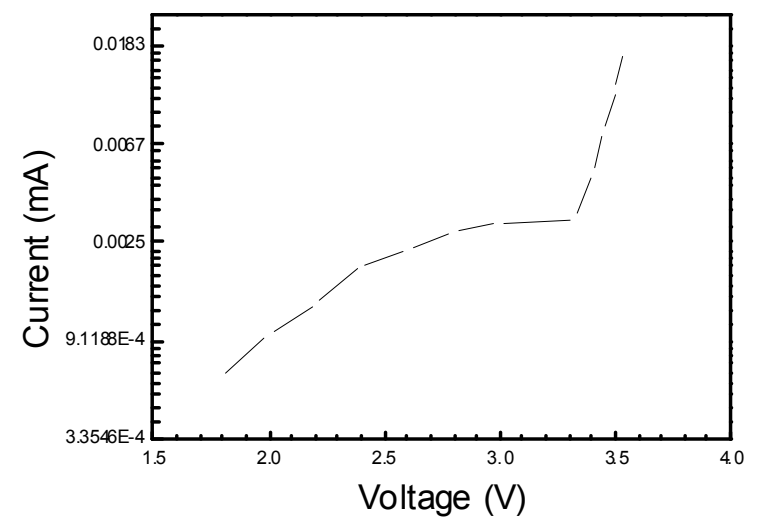

Fig.1 I-V plot ZnO thin film showing resistive switching

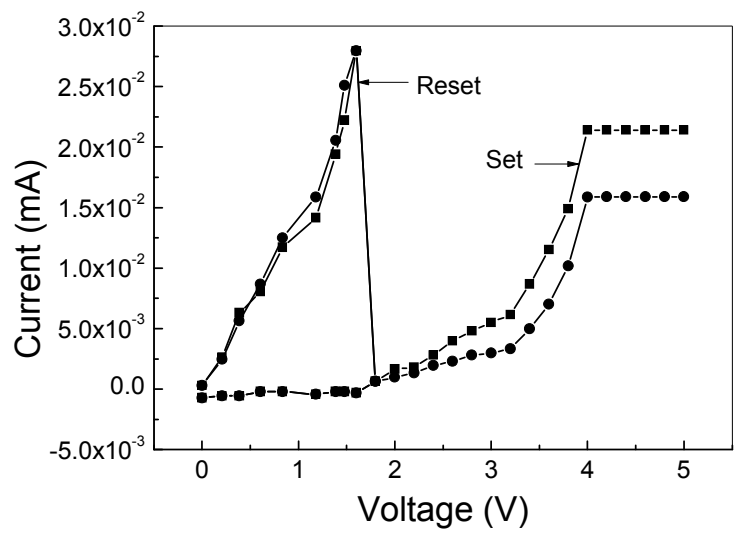

Fig. 2 Voltage-biased SET $I-V$ curve of memory switching with a current compliance of $1.6 \mathrm{~mA}$.

\section{DESIGNED WORK}

A Memory usually refers to semiconductor storage read-write random-access memory, typically DRAM (Dynamic-RAM). Memory can refer to other forms of fast but temporary storage. Storage refers to storage devices and their media not directly accessible by the CPU, typically hard disk drives, optical disc drives, and other devices slower than RAM but not volatile. 
One bit of memory. In dynamic RAM memory, a cell is made up of one transistor and one capacitor. In static RAM memory, a cell is made up of about five transistors.

Primary computer memory is made up of memory cells, where each memory cell contains exactly one number. Thus, a memory cell can be thought of as box into which a single number can be placed. The number contained in a memory cell can be changed over time. When a new number is stored into a memory cell, the old number contained in the memory cell is lost forever. At any time, the computer may peer into a memory cell to read the current contents of the memory cell. The computer may read the contents of a memory cell as many times as it wants, without disturbing it.

The idea behind the proposal is to use the $\mathrm{ZnO}$ thin film as a switching element instead of the transistor in a dynamic random access memory (DRAMs) cell. The DRAM cell consists the three main block: the array of 1-bit memory cell, the row decoder which select a single word line for a given n-bit row address a [1: $n]$, the column decoder which selects a single bit line for a given $\mathrm{m}$-bit column address $\mathrm{b}[1: \mathrm{m}]$ and route a 1-bit data to or from a selected memory cell. In a dynamic random access cell binary data is stored as a charge in a capacitor. Memory cell consists of a storage capacitor and a transistor as a switching element. In our proposed work the $\mathrm{ZnO}$ thin film is used as a switching element instead of the transistor. We use the $\mathrm{ZnO}$ semiconductor in the thin film form due to its unique properties. Figure 3 shows the typical memory organization. Figure 4 (a) shows the existing DRAM cell in which one transistor and one capacitor is used. In the existing DRAM cell transistor is used as a switching element. Figure 4 (b) shows the proposed idea in which we modify the memory (DRAM) cell with the $\mathrm{ZnO}$ thin film. In the $\mathrm{ZnO}$ thin film resistance switch between a high resistance HRS and low resistance state LRS which indicate the ON and OFF state and binary data is stored in a capacitor (memory cell) according to these states. The $\mathrm{ZnO}$ based dynamic memory cell exhibits resistive switching properties such as low switching voltage and switching cycling [12].

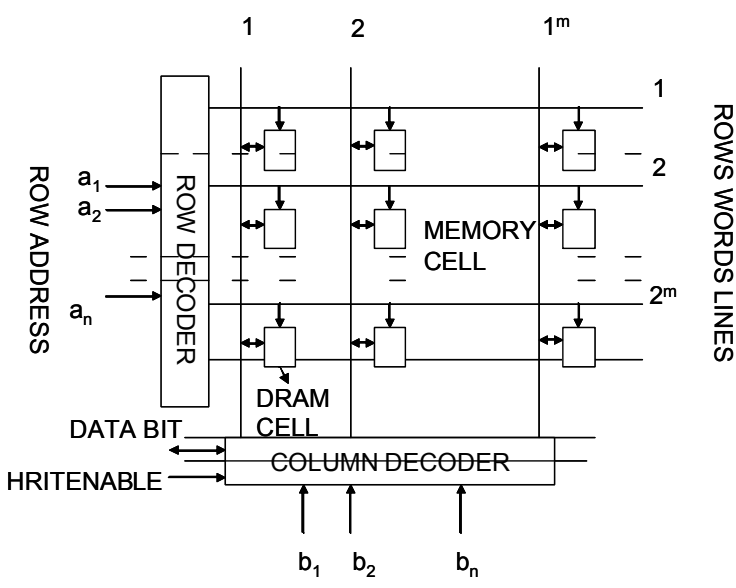

Fig. 3 Memory Organization

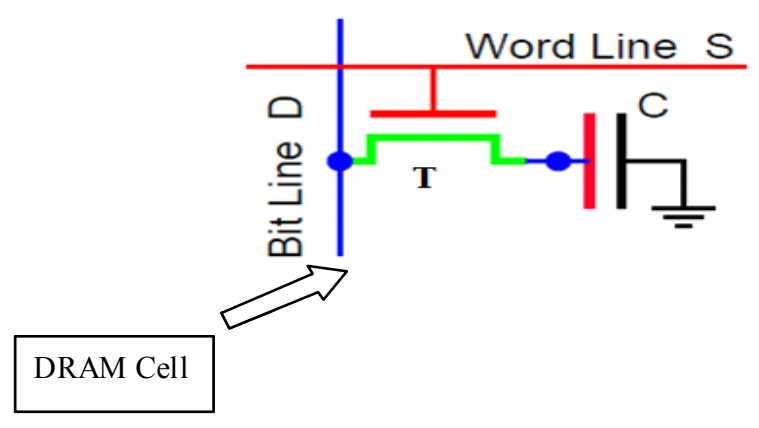

Fig. 4(a) Existing DRAM Cell

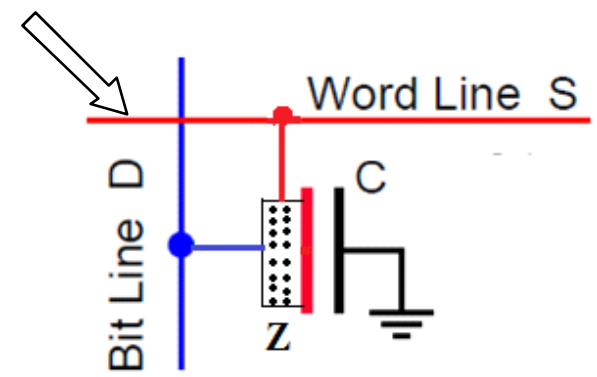

Fig. 4(b) Proposed DRAM Cell

\section{CONCLUSION}

This paper provides a brief knowledge about the $\mathrm{ZnO}$ thin film, an important part of nano technology and the brief idea about its use in dynamic random access memory cell. Their uses mainly include various storage purposes and as a resistance switching element. In our proposed work the dynamic memory cell work according to the resistance switching of the $\mathrm{ZnO}$ thin film so it provides better storage because it uses the lower operating voltage, faster switching rate, smaller and simpler cell structures.

\section{REFERENCES}

[1] K. M. Kim and C. S. Hwang, 2009. The conical shape filament growth model in unipolar resistance switching of $\mathrm{TiO}_{2}$ thin film. Appl. Phys. Lett. 94 (Mar. 2009) 122109-1122109-3.

[2] B. J. Choi, D. S. Jeong, S. K. Kim, C. Rohde, S. Choi, J. H. Oh, H. J. Kim, C. S. Hwang, K. Szot, R. Waser, B. Reichenberg, and S. Tiedke, 2005. Resistive switching mechanism of $\mathrm{TiO}_{2}$ thin films grown by atomic-layer deposition. J. Appl. Phys. 98 (Aug. 2005) 033715-1033715-10.

[3] R. Waser and M. Aono, 2007. Nanoionics-based resistive switching memories. Nat. Mater. 6 (2007) 833-840.

[4] J. J. Yang, F. Miao, M. D. Pickett, D. A. A. Ohlberg, D. R. Stewart, C. N. Lau, and R. Stanley Williams, 2009. The mechanism of electroforming of metal oxide memristive switches. Nanotechnology 20 (May 2009) 215201.

[5] D. B. Strukov, G. S. Snider, D. R. Stewart, and R. S. Williams, 2008. The missing memristor found. Nature 453(May2008) 80-83. 
[6] D. S. Jeong, H. Schroeder, and R. Waser, 2009. Mechanism for bipolar switching in a $\mathrm{Pt} / \mathrm{TiO}_{2} / \mathrm{Pt}$ resistive switching cell. Phys. Rev. B 79 (May 2009) 195317.

[7] W. Wang, S. Fujita, and S. S. Wong, 2009. RESET Mechanism of TiOx Resistance-Change Memory Device IEEE Electron Device Lett. 30 (July 2009) 733-735.

[8] A. Sawa, T. Fujii, M. Kawasaki, and Y. Tokura, 2004. Hysteretic current-voltage characteristics and resistance switching at a rectifying $\operatorname{Ti} \mathrm{Pr}_{0.7} \mathrm{Ca}_{0.3} \mathrm{MnO}_{3}$ interface. Appl. Phys. Lett. 85 (Sept. 2004) 4073-4075.

[9] J. Choi, J. Song, K. Jung, Y. Kim, H. Im, W. Jung, H. Kim, Y. H. Do, J. S. Kwak, and J. Hong, 2009. Bipolar resistance switching characteristics in a thin $\mathrm{Ti}-\mathrm{Ni}-\mathrm{O}$ compound film. Nanotechnology 20 (April 2009) 175704.

[10] K. M. Kim, B. J. Choi, Y. C. Shin, S. Choi, and C. S. Hwang, 2007. Anode-interface localized filamentary mechanism in resistive switching of $\mathrm{TiO}_{2}$ thin films Appl. Phys. Lett. 91 (July 2007) 012907-1-012907-3.

[12] W.-H. Tzeng, C.-W. Zhong, K.-C. Liu, K.-M. Chang, H.-C. Lin, Y.-C. Chan, C.-C. Kuo, F.-Y. Tsai, M. H. Tseng, P.-S. Chen, H.-Y. Lee, F. Chen and M.-J. Tsai, 2012. Resistive switching characteristics of multilayered $\left(\mathrm{HfO}_{2} / \mathrm{Al}_{2} \mathrm{O}_{3}\right)_{\mathrm{n}} \mathrm{n}$ $=19$ thin film. Thin Solid Films 520 (2012) 3415-3418.

[12] L. M. Kukreja, A. K. Das and P. Mishra, 2009. Studies on nonvolatile resistance memory switching in $\mathrm{ZnO}$ thin films. Bull. Mater. Sci. 32 (June 2009) 247-252.

[13] H. Shima, N. Zhong, and H. Akinaga, 2009. Switchable rectifier built with $\mathrm{Pt} / \mathrm{TiO}_{x} / \mathrm{Pt}$ trilayer. Appl. Phys. Lett. 94 (Feb. 2009) 082905-1-082905-3.

[14] C. Schindler, S. C. P. Thermadam, R. Waser, and M. N. Kozicki, 2007. Bipolar and Unipolar Resistive Switching in $\mathrm{Cu}$-Doped $\mathrm{SiO}_{2}$. IEEE Trans. Electron Devices 54 (Oct. 2007) 2762-2768.

[15] Y. Nakamura, T. Harada, H. Kuribara, A. Kishimoto, N. Motohira, and H. Yanagida, 1999. Nonlinear CurrentVoltage Characteristics with Negative Resistance Observed at $\mathrm{ZnO}-\mathrm{ZnO}$ Single-Contacts. J. Am. Ceram. Soc. 82 (1999) 3069-3074.

[16] J. W. Seo, S. J. Baik, S. J. Kang, Y. H. Hong, J. H. Yang, and K. S. Lim, 2011. A ZnO cross-bar array resistive random access memory stacked with heterostructure diodes for eliminating the sneak current effect Appl. Phys. Lett. 98 (June 2011) 233505-1-233505-3.

[17] J. Qiu, A. Shih, W. Zhou, Z. Mi, and I. Shih, 2011. Effects of metal contacts and dopants on the performance of $\mathrm{ZnO}$ based memristive devices. J. Appl. Phys. 110 (July 2011) 014513-1-014513-5.

[18] N. Fujimura, T. Nishihara, S. Goto and J. Xu, 1993. Control of preferred orientation for $\mathrm{ZnO}_{x}$ films: control of self-texture J. Cryst. Growth 130 (May 1993) 269-279.

[19] Y. E. Lee, Y. J. Kim and H. J. Kim, 1998. Thickness dependence of microstructural evolution of $\mathrm{ZnO}$ films deposited by rf magnetron sputtering. J. Mater. Res. 13 (1998) 1260-1265.

[20] C. Jin, R. Narayan, A. Tiwari, H. Zhou, A. Kvit and J. Narayan, 2005. Epitaxial growth of zinc oxide thin films on silicon. Materials Sci. Eng. (B), 117 (March 2005) 348354.
[21] S. O. Kucheyev, C. Jagadish, J. S. Williams, P. N. K. Deenapanray, M. Yano and K. Koike, 2003. Implant isolation of ZnO. J. Appl. Phys. 93 (2003) 2972-2976.

[22] E.M. Bachari, G. Baud, S. Ben Amor, M. Jacquet, 1999. Structural and optical properties of sputtered $\mathrm{ZnO}$ films. Thin Solid Films 348 (Jan. 1999) 165-172.

[23] W. Water, S.Y. Chu, 2002. Physical and structural properties of $\mathrm{ZnO}$ sputtered films. Mater. Lett. 55 (2002) $67-72$.

[24] J.J. Zhu, B.X. Lin, X.K. Sun, R. Yao, C.S. Shi, Z.X. Fu, 2005. Heteroepitaxy of $\mathrm{ZnO}$ film on $\mathrm{Si}$ (111) substrate using a 3C-SiC buffer layer. Thin Solid Films 478 (2005) 218-222.

[25] Y.I. Alivov, J.E.V. Nostrand, D.C. Look, M.V. Chukichev, B.M. Ataev, 2003. Observation of $430 \mathrm{~nm}$ electroluminescence from $\mathrm{ZnO} / \mathrm{GaN}$ heterojunction lightemitting diodes. Appl. Phys. Lett. 83 (2003) 2943-2945.

[26] N. Bouhssira, S. Abed, E. Tomasella, J. Cellier, A. Mosbah, M.S. Aida, M. Jacquet, 2006. Influence of annealing temperature on the properties of $\mathrm{ZnO}$ thin films deposited by thermal evaporation Appl. Surf. Sci. 252 (Jan. 2006) 5594-5597.

[27] Y. Zhang, B.X. Lin, X.K. Sun, Z.X. Fu, 2005. Temperature-dependent photoluminescence of nanocrystalline $\mathrm{ZnO}$ thin films grown on $\mathrm{Si}$ (100) substrates by the sol-gel process. Appl. Phys. Lett. 86 (March 2005) 131910-131912.

[28] T. Nakamura, Y. Yamada, T. Kusumori, H. Minoura, H. Mutoa, 2002. Improvement in the crystallinity of $\mathrm{ZnO}$ thin films by introduction of a buffer layer Thin Solid Films 411 (2002) 60-64.

[29] J.M. Bian, X.M. Li, C.Y. Zhang, L.D. Chen, Q. Yao, 2004 Synthesis and characterization of two-layer-structured $\mathrm{ZnO}$ $p-n$ homojunctions by ultrasonic spray pyrolysis Appl. Phys. Lett. 84 (April 2004) 3783-3785.

[30] R. Al Asmar, G. Ferblantier, F. Mailly, P. Gall- Borrut and A. Foucaran, 2004. Effect of annealing on the electrical and optical properties of electron beam evaporated $\mathrm{ZnO}$ thin films. Thin Solid Films 473 (2005) 49-53.

[31] B. J. Jin, S. H. Bae, S. Y. Lee and S. Im, 2000. Effects of native defects on optical and electrical properties of $\mathrm{ZnO}$ prepared by pulsed laser deposition. Mater Sci. Eng. (B) 71 (2000) 301-305.

[32] W. C. Shin and M. S. Wu, 1994. Growth of ZnO films on GaAs substrates with a $\mathrm{SiO}_{2}$ buffer layer by $\mathrm{RF}$ planar magnetron sputtering for surface acoustic wave applications. J. Cryst. Growth 137 (1994) 319-325.

[33] K. S. Weissemrider and J. Muller, 1997. Conductivity model for sputtered $\mathrm{ZnO}$-thin film gas sensors. Thin Solid Films 300 (1997) 30-41.

[34] M. S. Roman Chala, A. Rohatgi, W. B. Carter, J. P. Sharffer and T. K. Gupta, 1995. Photoluminescence study of $\mathrm{ZnO}$ varistor stability. J. Electron. Mater. 24 (1995) 413-419.

[35] A. Kumar, M. Kumar and B. P Singh, 2011. Nonvolatile resistance memory switching in polycrystalline $\mathrm{ZnO}$ thin films grown by RF magnetron sputtering. Int. J. Adv. Eng. Sci. \& Technol. 1 (2011) 118-122. 